\title{
Proceso educativo en programas de Arquitectura bajo el aislamiento preventivo obligatorio por causa del COVID-19
}

\section{Educational process of architecture programs in universities in Colombia under compulsory preventive isolation due to COVID-19}

\author{
Erika Tatiana Ayala-García \\ César Hernández-Suárez \\ Raúl Prada-Núñez \\ Universidad Francisco de Paula Santander, Cúcuta, Colombia
}

Open Access:

ISSN: $0124-2121$

E-ISSN: $2665-2420$

Artículo de Revisión Bibliográfica Copyright $\odot$ By Educación y Humanismo

Editor: Dhayana Fernández Matos Universidad Simón Bolívar

Correspondencia: César Hernández cesarauqusto@ufps.edu presenciales. Discusión y Conclusiones: como respuesta a la pandemia del COVID-19, los programas de Arquitectura en Colombia han debido realizar adaptaciones pedagógicas por medio de estrategias no presenciales tales como la utilización de plataformas virtuales y otros elementos tecnológicos.

Palabras clave: Aislamiento social, Arquitectura, Covid-19, Educación, Pandemia, Tecnología digital.

\begin{abstract}
Objective: this study aims to determine the effects of the transition from face-to-face to remote learning with the implementation of information and communication technologies (ICT) tools within architecture courses in universities in Colombia under two categories, namely, teachers and students. Method: the paper presents a descriptive, cross-sectional study that conducted a survey as the quantitative method. Results: the results highlighted that one of the challenges of institutions of higher education corresponds to the training of teachers and students in the use of ICT tools and platforms in the field of education, as well as to the formulation of strategies that lead to the improvement of non-attendance practices in education. Discussion and conclusion: in response to the COVID-19 pandemic, the architecture programs in universities in Colombia were forced to adapt their pedagogy using non-contact strategies, such as the use of virtual platforms and other technological elements.
\end{abstract}

Keywords: Architecture, COVID-19, Digital technology, Education, Pandemic, Social isolation. 


\section{Introducción}

El COVID- 19 causado por el virus SARS-CoV-2, fue declarado oficialmente como pandemia por la Organización Mundial de la Salud - OMS el 11 de marzo de 2020, como respuesta a sus altos niveles de propagación, de inacción y gravedad (Organización Mundial de la Salud - OMS, 2020a) (Mahase, 2020). Según el Coronavirus Disease (COVID-19) Dashboard en escala mundial con fecha del 20 de mayo de 2020 se han notificado ante la OMS 4.761.559 casos confirmados de COVID-19, y 317.529 muertes (OMS, 2020b). Para el caso de los países y los territorios de las Américas se han reportado para la misma fecha 2.105.801 casos acumulativos y 125.843 muertes (Pan American Health Organization PAHO, 2020), siendo Estados Unidos con 1.477 .459 el territorio con mayor número de casos confirmados, seguido de Rusia con 299,941 casos confirmados y Brasil 254.220, respectivamente (Organización Mundial de la Salud - OMS, 2020b). En el territorio colombiano, se registran para el 19 de mayo 17.687 casos confirmados y 630 muertes (Instituto Nacional de Salud Colombia, 2020).

Bajo este orden de ideas, el gobierno colombiano siendo consecuente con los impactos que ha generado la pandemia dentro del territorio, ha establecido una ruta legal contundente con el fin de desacelerar el proceso de propagación y mitigar los daños derivados de la pandemia, dentro de este proceso normativo se encuentran (ver tabla 1):

Tabla 1.

Normativa Covid-19 Colombia

$\begin{array}{cc}\begin{array}{c}\text { Normativa } \\ \text { Circular 017 (24 de } \\ \text { febrero 2020) }\end{array} & \begin{array}{c}\text { Emitida por } \\ \text { Ministerio del Trabajo }\end{array} \\ \begin{array}{c}\text { Resolución 385 (12 } \\ \text { marzo 2020) }\end{array} & \begin{array}{c}\text { Ministerio de Salud y } \\ \text { Protección social }\end{array} \\ \begin{array}{c}\text { Decreto 402 (13 de } \\ \text { marzo de 2020) }\end{array} & \text { Ministerio del Interior } \\ & \\ \text { Decreto 417 (17 } & \\ \text { marzo de 2020) } & \text { Presidencia de la } \\ \text { República } \\ \text { Circular 021 (17 de } \\ \text { marzo de 2020) }\end{array} \quad \begin{gathered}\text { Ministerio de } \\ \text { Educación Nacional }\end{gathered}$

Resolución 464 (18 de marzo de 2020)

Decreto 439 (20 de marzo de 2020)

Decreto457 (22 de marzo de 2020)
Ministerio de salud y protección social Ministerio de Transporte

Ministerio del Interior

\section{Decisiones Principales}

Lineamientos mínimos para implementar la promoción y prevención para preparación, respuesta y atención de casos de enfermedad por COVID-19.

Se declara la emergencia sanitaria por causa del coronavirus COVID-19 y se adoptan medidas para hacer frente al virus.

Cierre de frontera terrestre y fluvial con la República Bolivariana de Venezuela. Cierre de los pasos terrestres y fluviales autorizados de frontera con la República Bolivariana de Venezuela a partir del 14 de marzo de 2020 hasta el 30 de mayo de 2020.

Decreta el Estado de emergencia económica, social y ecológica por 30 días calendario.

Orientaciones para el desarrollo de procesos de planeación pedagógica y trabajo académico en casa como medida para la prevención de la propagación del COVID-19 así como para el manejo del personal docente, directivo docente y administrativo del sector de educación.

Se adopta medida sanitaria obligatoria de aislamiento preventivo para proteger adultos mayores de 70 años. Decreta suspender el desembarque con fines de ingreso o conexión en territorio colombiano, de pasajeros procedentes del exterior, por vía aérea.

Decreta aislamiento preventivo obligatorio de todas las personas habitantes de la República de Colombia con 
Decreto531 (8 de

abril de 2020)

\section{Decreto Legislativo}

538 (12 abril de 2020)

Decreto Legislativo

546 (14 abril de 2020)

Decreto 593 (24 de

abril de 2020)

Decreto 637(6 de mayo de 2020)
Ministerio del Interior

Ministerio de salud y protección social

Ministerio de Justicia y del Derecho.

Ministerio del Interior

Presidencia de la República. algunas excepciones desde el veinticinco (25) de marzo de 2020, hasta el trece (13) de abril de 2020.

Decreta aislamiento preventivo obligatorio de todas las personas habitantes de la República de Colombia con algunas excepciones desde 13 de abril de 2020, hasta el 27 de abril de 2020.

Decreta autorización a entidades de salud del orden territorial para adecuar sitios para la prestación de salud de manera temporal, Telesalud, llamado a talento Humano (en ejercicio o formación) para prestación de salud y declaración del COVID-19 como enfermedad laboral.

Decreta medidas de detención preventiva y de prisión domiciliaria transitorias, en el lugar de su residencia o en que el Juez autorice.

Decreta extensión del aislamiento preventivo obligatorio de todas las personas habitantes de la República de Colombia con algunas excepciones, a partir del $27 \mathrm{de}$ abril de 2020, hasta el 11 de mayo de 2020.

Decreta (prorroga) del Estado de emergencia económica, social y ecológica por 30 días calendario.

Fuente: Elaboración propia basada en la normativa expedida por el gobierno colombiano a partir del Covid-19 (2020).

Dentro de las recomendaciones y lineamientos estipulados en el orden mundial, la OMS ha establecido una clara diferenciación entre los procesos que deben regir las pautas de comportamiento de las personas a razón del COVID-19. Dentro de estas, precisa que se entiende por cuarentena la restricción de actividades y separación de personas que pueden haber estado expuestas al COVID-19. El aislamiento se establece como el procedimiento de separación de personas enfermas de COVID-19 y que pueden ser contagiosas y el distanciamiento físico corresponde a la recomendación de mantener distancia de al menos un metro entre personas (Organización Mundial de la Salud - OMS, 2020a) (González et ál., 2020).

Con respecto al ámbito académico, el 18 de marzo de 2020, la UNESCO estimó que 107 países habían aplicado cierres de instituciones educativas como medida de prevención ante el COVID-19, situación que afectó a millones de niños y jóvenes, correspondiente al 70\% de la población estudiantil mundial (UNESCO, 2020a). Los cierres de las instituciones educativas de orden preescolar, básica, media y superior se basan en el supuesto de que los brotes de gripe estacionaria (influenza) y coronavirus (Organización Panamericana de la Salud - OPS, 2020), pueden llegar a tener un índice de reducción e interrupción mediante la implementación del distanciamiento social entre los estudiantes (Sistema de vigilancia de la gripe en España - Instituto de Salud Carlos III, 16 de abril de 2018).

La eficacia de los cierres de las instituciones educativas y otras medidas de distanciamiento social tienen como fundamento que la transmisión de virus como la gripe tiende a ser impulsada por los contactos sociales de niños y adolescentes. Algunos estudios demuestran que los niños contribuyen más a la transmisión de la gripe que los adultos (Wallinga, Teunis, \& Kretzschmar, 2006). Por lo tanto, las medidas de cierre de las 
instituciones educativas actúan como una intervención no farmacéutica tendiente a la mitigación de las pandemias como la influenza, entre otras (Cauchemez et ál., 2009).

En el contexto nacional colombiano, se encuentra un informe de salud pública del Instituto Nacional de Salud (2007) que contiene información para controlar la transmisión de la influenza pandémica, aborda prácticas de distanciamiento social, y menciona el cierre de las instituciones educativas. Sin embargo, se debe reseñar que no se tienen evidencias sobre prácticas previas al año 2020 en este contexto, ya que no se había presentado una situación de tal magnitud como la que existe actualmente.

El distanciamiento social obligatorio llevado a cabo en gran parte de los países del mundo se centra en el cierre de las instituciones educativas y el traslado de las actividades laborales al perfil de trabajo en casa. Esto se ha configurado como un cambio radical a la cotidianidad de los núcleos familiares quienes ahora deben compartir desde casa actividades laborales y educativas; todo lo anterior con el fin de reducir el contacto y disminuir la proliferación del contagio del COVID-19. Sin embargo, luego de dos meses de implementación del aislamiento social obligatorio se empiezan a reconocer los efectos adversos producidos a partir de esta práctica, dentro de los más significativos se encuentran los perjuicios económicos derivados del cierre de establecimientos comerciales de pequeña, mediana y gran escala, así como los efectos negativos por los bajos niveles de productividad que trae como consecuencia una disminución de los ingresos por conceptos laborales. Por otra parte, perjuicios para el bienestar de las personas (haciendo un énfasis a las comunidades más vulnerables) en términos de necesidades básicas insatisfechas; así como efectos psicológicos negativos de los cuales se derivan el estrés postraumático, la confusión, el enojo, la frustración, y el estigma (Brooks et al., 2020).

Con respecto al ámbito educativo, se destacan los perjuicios para los niños, niñas, adolescentes y docentes a raíz del traslado a la educación no presencial, que puede significar a corto plazo la ampliación de las brechas educativas (UNICEF, 2020; Leybold-Johnson, 2020) como respuesta a factores como la interrupción del aprendizaje, la falta de preparación y conocimiento de prácticas asociadas a la educación a distancia, la desigualdad en el acceso al aprendizaje digital, la desigualdad y el acceso a internet; y por ende el incremento de las tasas de deserción escolar (UNESCO, 2020b); entre otras.

Es de resaltar, además, que como consecuencia de la emergencia mundial, el factor educativo ha debido realizar cambios significativos dentro de las dinámicas propias de la enseñanza y el aprendizaje, sorteando así no sólo el desafío de mantener su comunidad sana (libre de COVID-19) sino la necesidad de implementar medidas que garanticen el vínculo estudiante-docente y familia-escuela; la entrega de contenido alineado al currículo escolar y el acompañamiento y monitoreo del proceso de aprendizaje (Vásquez et ál., 2020). Lo anterior, teniendo en cuenta el panorama real de las instituciones de educación y los núcleos familiares, en donde las soluciones adoptadas dependen de las capacidades prexistentes de infraestructura y de los contenidos disponibles de las instituciones 
educativas para armar un modelo rápido que responda a la educación desde el hogar.

Bajo este orden de ideas, se debe reseñar que el distanciamiento social provocado por la pandemia del COVID-19, ha generado cambios abruptos en las rutinas y los hábitos de las personas. En el proceso educativo particularmente, los estudiantes y docentes han experimentado un cambio significativo al tener que realizar una adaptación rápida a la educación no presencial, bajo el soporte de la tecnología y otros medios, que ha configurado en un plazo corto metas y desafíos de alto alcance e impacto asociadas a garantizar la continuidad de las clases durante el aislamiento. Hecho que a su vez presupone interrogantes como ¿Cuáles son los efectos que ha tenido el aislamiento en estudiantes y docentes de los programas de Arquitectura en Colombia? ¿Existen diferencias en las habilidades y destreza en el uso de las TIC y en el ámbito de las relaciones sociales o en el ámbito académico?

\section{COVID-19, Educación Superior y Arquitectura}

Debido al cumplimiento de los lineamientos establecidos por la UNESCO en los que se recomienda cancelar o posponer programas de intercambio, de salida o entrada a nivel internacional, cancelar reuniones y encuentros de carácter académico, suspender actividades académicas presenciales, precisar mecanismos que promuevan los sistemas de información y comunicación en remoto, utilizar plataformas de aprendizaje en línea, así como fortalecer los campus virtuales (Instituto Internacional para la Educación Superior en América Latina y el Caribe - IESALC, 2 de abril 2020). Además de lo establecido en la Circular 021 del 17 de marzo de 2020 emitida por el Ministerio de Educación Nacional colombiano en donde se condensan las orientaciones para el desarrollo de procesos de planeación pedagógica y trabajo académico en casa como medida para la prevención de la propagación del COVID-19, y el manejo del personal docente, directivo docente y administrativo del sector de educación, se ha generado un cambio en la modalidad de la educación en las Instituciones de Educación Superior (en adelante IES). Esto debido a que, las IES se han visto en la necesidad de ampliar y sumar estrategias en materia pedagógica a través de las cuales se pueda garantizar la prestación del servicio educativo, aún en época de pandemia, mediante el fortalecimiento de la infraestructura tecnológica e informática que le permita a docentes y estudiantes continuar con el desarrollo de su semestre académico, trasladando así una educación centrada en un proceso presencial mediante el desarrollo de asignaturas prácticas, teóricas y teórico-prácticas, a procesos no presenciales apoyados en las TIC y en estrategias pedagógicas más flexibles que permitan dar cumplimiento bajo aislamiento obligatorio a los programas establecidos para el primer semestre académico del 2020.

Para el caso específico de Colombia, Saldarriaga (1996) ha enfatizado que la Arquitectura se caracteriza por no ser una disciplina autónoma, contrario esto, su quehacer disciplinar y académico se encuentra influenciado por el medio natural y cultural propio del contexto en el que se desarrolla. Dentro de ella, convergen conocimientos relacionados con áreas de formación centradas en el abordaje de temáticas como el espacio, la técnica, la 
representación, lo humano, la historia, y el diseño; entre otros (Universidad Francisco de Paula Santander - UFPS, 2018) que, de acuerdo a la Ley 1324 de 2009, son evaluados por el Instituto Colombiano para la Educación - ICFES mediante las pruebas Saber Pro, a través del módulo de proyecto de Arquitectura que comprende los componentes urbano-ambiental, formal-espacial, tecnológico, funcional y comunicativo (Instituto Colombiano para la Evaluación de la Educación - ICFES, 2019a; 2019b); así como por el módulo genérico y el específico para los programas de Arquitectura (ICFES, 2019c).

Con respecto a la enseñanza dentro de los programas de Arquitectura a nivel nacional, es pertinente reseñar que dentro de las mallas curriculares se destaca que la asignatura denominada como "Taller", "Proyecto" o "Talleres de diseño" lidera el componente proyectual. A través de esta asignatura de tipo "práctica", el estudiante canaliza las áreas de conocimiento disciplinar como la expresión visual, la técnica, el urbanismo, el diseño, la teoría e historia y lo socio- humanístico (Universidad Francisco de Paula Santander - UFPS, 2018), junto con el componente tecnológico, urbano territorial, y de representación (Saldarriaga, Mateus y Pinzón, 2011). Dentro de los talleres de diseño se realizan prácticas, salidas de campo o de reconocimiento de sectores de estudio, ejercicios rápidos, construcción soluciones a problemáticas a escala macro, meso y micro de la ciudad a través de propuestas grupales e individuales, bajo el asesoramiento constante y presencial del director de curso. Así mismo, los pensum de Arquitectura cuentan con asignaturas de tipo teórico y teórico-práctico, en las cuales los estudiantes abordan por medio de trabajo presencial e independiente los aspectos asociados a los componentes de la técnica, la representación, socio-humanístico y de la teoría y la historia, según corresponda al pensum del programa de Arquitectura de cada universidad. Lo anteriormente expuesto, deja en evidencia que las herramientas pedagógicas propias de la presencialidad han marcado a lo largo del tiempo los parámetros de ejecución y desarrollo de las actividades académicas en los programas de Arquitectura en el orden nacional.

El panorama de la educación bajo una figura no presencial establece dentro de los programas de Arquitectura en Colombia la necesidad de generar cambios dentro del desarrollo de su práctica pedagógica, debido que se prevé una intersección entre las TIC, la educación y la pedagogía en Colombia (Parra, 2010). Bajo este orden de ideas, desde las IES se ha establecido un triple reto, que consiste en una primera medida en establecer parámetros y lineamientos claros asociados a rutas estratégicas con el fin de dar cumplimiento al primer semestre académico del 2020. Generalmente, a nivel nacional esto se ha realizado simultáneamente bajo la puesta en marcha de ciclo de capacitaciones rápidas e intensivas sobre el uso de herramientas TIC para docentes, ya que éstos deben poseer competencias TIC (Hernández, Arévalo y Gamboa, 2016; Hernández, Gamboa y Ayala, 2016; Gamboa, Hernández y Prada, 2018; Hernández, Prada y Ramírez, 2018).

Por otro lado, el segundo reto que se asume a raíz del cambio a la modalidad no presencial, corresponde a los docentes, quienes mientras deben atender los nuevos procesos y la adaptación de sus cátedras a esta modalidad, deben asistir a las capacitaciones 
sobre TIC y poner en práctica estos nuevos conocimientos dentro de la planeación estratégica de las materias a su cargo, para construir ambientes innovadores seleccionando las estrategias y las TIC adecuadas para que sus estudiantes logren sus objetivos de aprendizaje (Martín, Hernández, y Mendoza, 2017).

Es necesario aclarar que dentro de la Arquitectura las asignaturas de denominación práctica han requerido una mayor precisión con respecto a la adaptación a la educación no presencial, al tener en cuenta que se deben reorganizar los métodos y la metodología utilizada dentro de la misma, sobre todo al tener en cuenta que dentro de los programas de Arquitectura existe la posibilidad de llevar a cabo procesos de diseño análogos o digitales que se materializan de acuerdo a las competencias propias de los estudiantes. Sin embargo, se debe precisar que dentro de las asignaturas teóricas o teórico - prácticas, los docentes también han tenido que implementar múltiples herramientas asociadas a las TIC con el fin de dar cumplimiento a la transmisión y generación de conocimiento y por ende la adquisición de competencias por parte de los estudiantes. En palabras de Aparici y Silva (2012, p. 52), podría deberse a una "lógica de la distribución, propia de los medios de comunicación de masas y subutiliza las potencialidades comunicativas que ofrece la web. Por otra parte, las redes sociales como Twitter, Instagram o en el mejor de los casos como "..., Facebook, que se ha convertido en el entorno central de comunicación y entretenimiento..." (Linne, 2014, p.193).

El tercer reto que asumen las IES, corresponde al estamento estudiantil, teniendo en cuenta que el proceso de cambio a modalidad no presencial a razón del COVID-19 se rige a partir del proceso de adaptación a las nuevas formas de enseñanza y evaluación. Con respecto a esto, la Dirección de Comunicaciones de la Fundación Universitaria del Área Andina (2020) asegura que "La transición trae retos para quienes no están preparados y tanto profesores como estudiantes deben sacar provecho de todo el potencial que trae consigo el mundo digital (...) Por eso, entre los retos que existen en el país para hacer la migración a la virtualidad está la necesidad de cubrir esta brecha digital".

\section{Método}

\section{Tipo de investigación}

La investigación utilizó una metodología cuantitativa a nivel transversal descriptivo (Hernández, Fernández y Baptista, 2010) puesto que los datos son recolectados de la fuente primaria sin la manipulación de variables en un único momento específico de tiempo a los cincuenta días de iniciado el aislamiento obligatorio preventivo en Colombia. Se recurre a la estadística descriptiva para identificar porcentajes o frecuencias de las variables objeto de estudio. 


\section{Población y Muestra}

La población está integrada por el grupo de estudiantes y docentes que laboran en el programa de Arquitectura en las diversas IES en Colombia. Se recurre al muestreo no probabilístico como técnica para la selección de la muestra. Para el desarrollo de esta investigación se envió vía correo electrónico la invitación a diligenciar la encuesta, de esta manera la muestra está conformada por los docentes y estudiantes de Arquitectura que accedieron y dieron cumplimiento al diligenciamiento de la encuesta por medio de 406 encuestas diligenciadas durante un periodo de 7 días.

\section{Instrumento para recolección de datos}

Para la recolección de datos se recurrió como instrumento a la encuesta, la cual estaba compuesta de 23 ítems distribuidos en cuatro variables descritos en la tabla 2.

Tabla 2.

Descripción de variables

\begin{tabular}{|c|c|c|}
\hline Variable & Descripción & Indicadores \\
\hline Perfil demográfico & $\begin{array}{l}\text { Corresponde a aquellas } \\
\text { características propias del } \\
\text { informante }\end{array}$ & $\begin{array}{l}\text { Género, edad, estado civil, rol que } \\
\text { desempeña, institución educativa } \\
\text { donde estudia o labora, semestre } \\
\text { académico y actividad principal. }\end{array}$ \\
\hline $\begin{array}{c}\text { Competencias TIC con uso } \\
\text { social }\end{array}$ & $\begin{array}{c}\text { Agrupa todos los ítems que } \\
\text { pretenden caracterizar la } \\
\text { incorporación de los recursos TIC } \\
\text { en la vida social del informante }\end{array}$ & $\begin{array}{l}\text { Desempeño en redes sociales, } \\
\text { capacitación, tiempo diario dedicado a } \\
\text { ellas, uso de las redes sociales, red } \\
\text { social con mayor frecuencia utilizada }\end{array}$ \\
\hline $\begin{array}{c}\text { Competencias TIC con uso } \\
\text { académico }\end{array}$ & $\begin{array}{c}\text { Agrupa todos los ítems que buscan } \\
\text { caracterizar la incorporación de los } \\
\text { recursos TIC en el proceso de } \\
\text { enseñanza }\end{array}$ & $\begin{array}{c}\text { Continuidad del semestre académico, } \\
\text { uso de clases no presenciales antes } \\
\text { del aislamiento, herramientas } \\
\text { utilizadas en las clases, nivel de } \\
\text { desempeño, tiempo diario dedicado, } \\
\text { dificultades, cursos de capacitación y } \\
\text { efectos }\end{array}$ \\
\hline Afectación emocional & $\begin{array}{l}\text { Posibles efectos derivados del } \\
\text { aislamiento obligatorio que afectan } \\
\text { la parte emocional del informante }\end{array}$ & $\begin{array}{l}\text { Estrés, desilusión, culpa, } \\
\text { concentración, sueño, llanto, tensión, } \\
\text { interés, indecisión, nivel de energía, } \\
\text { irritabilidad, apetito y cansancio }\end{array}$ \\
\hline
\end{tabular}

Fuente: Elaboración propia a partir del instrumento de encuesta realizado para la investigación.

Dado que el instrumento fue propuesto por el grupo investigador, se recurrió a la validación de contenido por juicio de expertos (Summers, 1976). Se conformó un panel de nueve personas, tres arquitectos en ejercicio que desempeñan funciones docentes, tres estudiantes, dos directores de programa académico y un experto en estadística. Durante tres sesiones de dos horas cada una, los participantes fueron analizando las variables a medir y los indicadores en cada una de ellas, revisando la redacción de cada ítem junto con las posibles escalas de respuesta. 


\section{Procedimiento y análisis de datos}

Para la recolección de los datos se contó con la colaboración de los directores del programa de Arquitectura de diversas universidades del país, quienes compartieron vía correo electrónico el link de la encuesta entre sus docentes y estudiantes, informándoles el propósito de la investigación e invitándoles a diligenciarlo de forma voluntaria. El diligenciamiento de la encuesta corresponde a una duración promedio de seis minutos. Los datos fueron descargados en un archivo Excel para luego ser exportados al SPSS $\vee 25$, software con el que se realizaron los respectivos análisis descriptivos.

\section{Resultados}

Como ya se mencionó el instrumento estaba compuesto de tres secciones, las cuales fueron diligenciadas por un total de 406 personas (271 estudiantes y 135 docentes) las cuales serán analizadas descriptivamente a continuación por separado, ello con el fin de determinar el efecto del aislamiento en cada población.

\section{Estudiantes}

\section{Perfil demográfico}

En la tabla 3 se organizaron las características demográficas de los estudiantes que participaron en el estudio. Se puede observar que en cuanto al sexo están distribuidos en partes iguales con un ligero predominio de los hombres, con edades que oscilan entre $15 \mathrm{y}$ 29 años; siendo solteros en aproximadamente el $95 \%$ de los casos. Respecto a la institución educativa en dónde adelantan sus estudios de Arquitectura se contó con la participación de seis instituciones educativas distribuidas a lo largo de la región Andina del país (Pasto, Armenia, Manizales, Bogotá, Tunja, Bucaramanga, Pamplona y Cúcuta). Finalmente, respecto al semestre que cursan actualmente, el $52 \%$ de ellos han superado el quinto semestre, pero se logró obtener participación de los diez semestres del ciclo de formación profesional.

Tabla 3.

Perfil demográfico de los estudiantes informantes

\begin{tabular}{cccc}
\hline Característica & Niveles de respuesta & Frecuencia & Porcentaje \\
\hline \multirow{2}{*}{ Género } & Masculino & 136 & $50,2 \%$ \\
\cline { 2 - 4 } & Femenino & 135 & $49,8 \%$ \\
\cline { 2 - 4 } & Total & $\mathbf{2 7 1}$ & $\mathbf{1 0 0 , 0} \mathbf{0}$ \\
\hline \multirow{2}{*}{ Edad } & Entre 30 y 40 años & 10 & $3,7 \%$ \\
\cline { 2 - 4 } & Entre 20 y 29 años & 193 & $71,2 \%$ \\
\cline { 2 - 4 } & Menos de 20 años & 68 & $25,1 \%$ \\
\cline { 2 - 4 } & Total & $\mathbf{2 7 1}$ & $\mathbf{1 0 0 , 0 \%}$ \\
\hline Estado Civil & Soltero(a) & 257 & $94,8 \%$ \\
& Casado(a) & 2 & $0,7 \%$ \\
\hline
\end{tabular}




\begin{tabular}{|c|c|c|c|}
\hline & Unión libre & 10 & $3,7 \%$ \\
\hline & Separado(a) & 2 & $0,7 \%$ \\
\hline & Total & 271 & $100,0 \%$ \\
\hline \multirow{7}{*}{$\begin{array}{c}\text { Actualmente } \\
\text { estudia en }\end{array}$} & Institución Universitaria CESMAG & 25 & $9,2 \%$ \\
\hline & Universidad Francisco de Paula Santander & 91 & $33,7 \%$ \\
\hline & Universidad de Pamplona & 13 & $4,8 \%$ \\
\hline & Universidad la Gran Colombia & 73 & $26,9 \%$ \\
\hline & Universidad Nacional de Colombia & 15 & $5,5 \%$ \\
\hline & Universidad Santo Tomás de Aquino & 54 & $19,1 \%$ \\
\hline & Total & 271 & $100,0 \%$ \\
\hline \multirow{11}{*}{$\begin{array}{c}\text { ¿Qué semestre } \\
\text { cursa } \\
\text { actualmente? }\end{array}$} & Primero & 31 & $11,4 \%$ \\
\hline & Segundo & 24 & $8,9 \%$ \\
\hline & Tercero & 37 & $13,7 \%$ \\
\hline & Cuarto & 15 & $5,5 \%$ \\
\hline & Quinto & 24 & $8,9 \%$ \\
\hline & Sexto & 17 & $6,3 \%$ \\
\hline & Séptimo & 38 & $14,0 \%$ \\
\hline & Octavo & 31 & $11,4 \%$ \\
\hline & Noveno & 39 & $14,4 \%$ \\
\hline & Décimo & 15 & $5,5 \%$ \\
\hline & Total & 271 & $100,0 \%$ \\
\hline
\end{tabular}

Fuente: Elaboración propia a partir del instrumento de Encuesta realizado para la investigación (2020).

\section{Competencias TIC con uso social}

En esta categoría se pretende establecer el nivel de dominio que poseen los informantes sobre diversas herramientas TIC, analizadas desde su uso social. De esta manera, se determinó que con respecto al uso y desempeño en las diversas redes sociales el 93,4\% de los encuestados afirmo que su desempeño es adecuado y de ellos se destacan $56,1 \%$ quienes afirman que es bueno.

El 79,7\% afirman nunca haber hecho cursos o haber recibido capacitación formal (por parte de una institución o instructor) para el manejo de herramientas o plataformas virtuales, luego su conocimiento es empírico, heredado principalmente de la interacción con su círculo de amigos y familiares.

Con respecto a la frecuencia de uso diario que dedican a las redes, se pudo determinar que el $97,8 \%$ del grupo de estudiantes informantes destina tiempo para esa actividad, destacando que de $37,6 \%$ dedican cuando mucho dos horas diarias mientras que el porcentaje restante dedican más de dos horas. Bajo el cuestionamiento, "Especifique la razón por las que utiliza las redes sociales" el 59,8\% de los informantes respondió que se dedicaban al desarrollo de actividades asociadas al ocio, entre tanto el porcentaje restante aseguró que invierte ese tiempo en la búsqueda de noticias con el fin de estar informados. Es de resaltar, que el uso excesivo de tiempo dedicado a esta actividad puede afectar el cumplimiento de deberes académicos. 
Finalmente, al indagar sobre la red social utilizada con mayor frecuencia se pudo identificar que Instagram, Facebook, Tinder y Twitter concentran el 99,6\% de los casos, con el $48,0 \%, 35,4 \%, 11,1 \%$ y $5,2 \%$ respectivamente. En la tabla 4 se pueden corroborar los principales hallazgos de esta categoría.

Tabla 4.

Tabla cruzada entre el tiempo diario dedicado a las redes sociales vs Principal razón por la que las utiliza y la red social más utilizada

\begin{tabular}{|c|c|c|c|c|c|c|}
\hline \multirow{4}{*}{$\begin{array}{c} \\
\text { Principal } \\
\text { razón por la } \\
\text { que utiliza las } \\
\text { redes sociales }\end{array}$} & \multicolumn{6}{|c|}{$\begin{array}{c}\text { ¿Con qué frecuencia diaria utiliza las } \\
\text { redes sociales? }\end{array}$} \\
\hline & & $\begin{array}{c}\text { Dos } \\
\text { horas o } \\
\text { menos }\end{array}$ & $\begin{array}{l}\text { Entre dos y } \\
\text { cinco horas }\end{array}$ & $\begin{array}{l}\text { Más de } \\
\text { cinco } \\
\text { horas }\end{array}$ & $\begin{array}{c}\text { No } \\
\text { utilizo }\end{array}$ & Total \\
\hline & Ocio & $20,3 \%$ & $19,6 \%$ & $18,5 \%$ & $1,5 \%$ & $59,8 \%$ \\
\hline & $\begin{array}{c}\text { Para estar } \\
\text { enterado de los } \\
\text { últimos } \\
\text { acontecimientos }\end{array}$ & $17,3 \%$ & $15,1 \%$ & $7,0 \%$ & $0,7 \%$ & $40,2 \%$ \\
\hline & Total & $37,6 \%$ & $34,7 \%$ & $25,5 \%$ & $2,2 \%$ & $100,0 \%$ \\
\hline \multirow{6}{*}{$\begin{array}{l}\text { ¿Cuál es la red } \\
\text { social que } \\
\text { utiliza con } \\
\text { mayor } \\
\text { frecuencia? }\end{array}$} & Facebook & $16,6 \%$ & $10,0 \%$ & $8,1 \%$ & $0,7 \%$ & $35,4 \%$ \\
\hline & Instagram & $14,0 \%$ & $19,9 \%$ & $13,7 \%$ & $0,4 \%$ & $48,0 \%$ \\
\hline & Tinder & $3,3 \%$ & $3,7 \%$ & $3,3 \%$ & $0,7 \%$ & $11,1 \%$ \\
\hline & Twitter & $3,7 \%$ & $1,1 \%$ & $0,4 \%$ & $0,0 \%$ & $5,2 \%$ \\
\hline & Otra & $0,0 \%$ & $0,0 \%$ & $0,0 \%$ & $0,4 \%$ & $0,4 \%$ \\
\hline & Total & $37,6 \%$ & $34,7 \%$ & $25,5 \%$ & $2,2 \%$ & $100,0 \%$ \\
\hline
\end{tabular}

Fuente: Elaboración propia a partir del instrumento de encuesta realizado para la investigación (2020).

\section{Competencias TIC con uso académico}

Esta categoría surge como complemento a la anterior, y busca precisar el nivel de apropiación que poseen los informantes sobre las diversas herramientas TIC desde su incorporación al proceso de enseñanza. Se indagó si actualmente estaban desarrollando su semestre bajo la modalidad no presencial con ayuda de recursos digitales, a lo que el $98,2 \%$ de los informantes respondió que "Si". Se resalta que casi la totalidad de estudiantes encuestados han estado desarrollando sus diversos cursos en modalidad no presencial, pero el $80,4 \%$ de ellos afirman que nunca habían participado de este tipo de actividades virtuales.

Al indagar sobre las diversas plataformas o herramientas que se han implementado como recurso de enseñanza los informantes podían seleccionar cuántas han utilizado. Esto permitió complementar un total de 957 opiniones, a partir de las cuales se logró determinar que el $93 \%$ se concentran en seis aplicaciones, de las cuales el $64 \%$ son aplicaciones sincrónicas (Hangouts Meet, WhatsApp, Zoom y Google Classroom) orientadas al desarrollo de conferencias o reuniones grupales en las que se pueden incluir audio y/o video, al tiempo que opcionalmente admiten la grabación de la misma (para aquellos que presentan dificultades de acceso), mientras que un $29 \%$ son aplicaciones que permiten el envío y 
almacenamiento de archivos (con limitaciones de capacidad y cantidad) en actividades asincrónicas.

Tabla 5.

Relación de herramientas o plataformas implementadas en el trabajo no presencial

\begin{tabular}{ccc}
\hline Plataforma o herramienta & Frecuencia & Porcentaje \\
\hline Hangouts Meet & 187 & $20 \%$ \\
WhatsApp & 174 & $18 \%$ \\
Correoelectrónico & 156 & $16 \%$ \\
Zoom & 154 & $16 \%$ \\
Plataforma Institucional & 124 & $13 \%$ \\
Google Classroom & 96 & $10 \%$ \\
Microsoft Teams & 50 & $5 \%$ \\
Otra & 16 & $\mathbf{2 \%}$ \\
\hline Total & $\mathbf{9 5 7}$ & $\mathbf{1 0 0} \%$
\end{tabular}

Fuente: Elaboración propia a partir del instrumento de Encuesta realizado para la investigación (2020).

En la tabla 6 permite identificar un grupo de informantes (17,3\%) que afirman que su desempeño en el manejo de estas herramientas digitales es deficiente, siendo esta la causa por la cual no participan en actividades académicas en el 2,9\% de los casos. Se destaca el grupo de informantes (equivalente al 22,9\%) que afirman dedicarle sólo dos horas o menos al trabajo en las plataformas virtuales para el cumplimiento de deberes académicos, tiempo que puede resultar insuficiente para el mismo bajo los esquemas de enseñanza propios de la Arquitectura. Finalmente, el 73,1\% mencionan dedicarle más de dos horas al día en el uso de estos recursos digitales y de ellos el $38,4 \%$ dicen tener un desempeño bueno.

Tabla 6.

Tabla cruzada entre el tiempo diario dedicado a las plataformas vs su valoración de desempeño en las mismas

¿Con qué frecuencia diaria utiliza las plataformas virtuales con fines educativos?

\begin{tabular}{|c|c|c|c|c|c|c|}
\hline & & & \multirow[b]{2}{*}{ Total } \\
\hline & & $\begin{array}{l}\text { Dos horas } \\
\text { o menos }\end{array}$ & $\begin{array}{l}\text { Entre dos y } \\
\text { cinco horas }\end{array}$ & $\begin{array}{c}\text { Más de cinco } \\
\text { horas }\end{array}$ & $\begin{array}{c}\text { No } \\
\text { utilizo }\end{array}$ & \\
\hline \multirow{4}{*}{$\begin{array}{c}\text { ¿Considera que } \\
\text { su desempeño } \\
\text { en el manejo de } \\
\text { herramientas o } \\
\text { plataformas } \\
\text { virtuales con } \\
\text { fines educativo } \\
\text { es? }\end{array}$} & Bueno & $8,1 \%$ & $14,4 \%$ & $24,0 \%$ & $0,4 \%$ & $46,8 \%$ \\
\hline & $\begin{array}{c}\text { Acepta } \\
\text { ble }\end{array}$ & $10,0 \%$ & $12,9 \%$ & $12,2 \%$ & $0,7 \%$ & $35,8 \%$ \\
\hline & $\begin{array}{c}\text { Deficie } \\
\text { nte }\end{array}$ & $4,8 \%$ & $3,7 \%$ & $5,9 \%$ & $2,9 \%$ & $17,3 \%$ \\
\hline & Total & $22,9 \%$ & $31,0 \%$ & $42,1 \%$ & $4,1 \%$ & $100,0 \%$ \\
\hline
\end{tabular}

Fuente: Elaboración propia a partir del instrumento de Encuesta realizado para la investigación (2020).

La información reseñada en la tabla anterior pone en evidencia la necesidad del uso de estos recursos en estos momentos de aislamiento preventivo obligatorio como herramienta alterna para el cumplimiento de los compromisos académicos. A partir de esta consideración 
se establece el cuestionamiento, ¿presenta dificultad en el manejo de estas herramientas con fines educativos? Ante lo cual se determinó que sólo el 39,1\% dan respuesta negativa, mientras que el 49,8\% dice que ha presentado dificultades tales como caídas del sistema, saturación de la red, carencia de internet, entre muchos otros.

El 87,8\% afirman que nunca han recibido capacitación por parte de sus docentes o instituciones educativas en dónde estudian, sobre el uso o manejo de estos recursos digitales con fines educativos; por lo que el $75,3 \%$ de ellos manifiestan que estos procesos de virtualización de la educación han afectado de forma negativa su rendimiento académico, debido a que en el $91,9 \%$ de los casos el docente considera muy importante que el estudiante asista a las actividades de orden no presencial. Este panorama puede resultar medianamente alentador para aquellos estudiantes a quienes les han ofrecido la alternativa de trabajo asincrónico (equivalente al 8,1\%), sin embargo, presenta como dificultad la demora de respuesta del docente en caso de existencia de dudas.

Al pedirle a los informantes que califiquen su rendimiento académico en este período de aislamientos obligatorio, se determina que el $42,8 \%$ de ellos afirman que ha tenido resultados favorables, en contraste con el porcentaje restante $(57,2 \%)$ quienes dicen haber tenido dificultades académicas, destacando que en el 16,3\% de ellos la situación es muy crítica.

\section{Afectación emocional}

Por medio de esta categoría se determinó el efecto emocional que ha propiciado en los estudiantes el aislamiento preventivo obligatorio, el cual ha forzado el cambio radical de los hábitos de vida.

Tabla 7.

Relación del grado de afectación emocional derivado del aislamiento preventivo obligatorio

Nivel de afectación

\begin{tabular}{cccc}
\hline Sentimientos & $\begin{array}{c}\text { Ha } \\
\text { reducido }\end{array}$ & $\begin{array}{c}\text { Se mantiene } \\
\text { igual }\end{array}$ & $\begin{array}{c}\text { Ha } \\
\text { aumentado }\end{array}$ \\
\hline Estrés & $8,5 \%$ & $21,4 \%$ & $70,1 \%$ \\
\hline Desilusión & $13,7 \%$ & $32,5 \%$ & $53,9 \%$ \\
\hline Disconformidad consigo mismo & $20,7 \%$ & $36,9 \%$ & $42,4 \%$ \\
\hline Sentimiento de culpa & $28,4 \%$ & $46,9 \%$ & $24,7 \%$ \\
\hline Nivel de concentración & $62,7 \%$ & $22,9 \%$ & $14,4 \%$ \\
\hline Hábitos de sueño & $40,6 \%$ & $22,5 \%$ & $36,9 \%$ \\
\hline Llanto & $28,0 \%$ & $47,2 \%$ & $24,7 \%$ \\
\hline Agitación o tensión & $17,3 \%$ & $30,3 \%$ & $52,4 \%$ \\
\hline Interés por realizar actividades & $42,4 \%$ & $33,6 \%$ & $24,0 \%$ \\
\hline Indecisión & $12,9 \%$ & $42,1 \%$ & $45,0 \%$ \\
\hline de actividades & $59,8 \%$ & $29,9 \%$ & $10,3 \%$
\end{tabular}




\begin{tabular}{clll}
\hline Irritabilidad & $10,3 \%$ & $26,9 \%$ & $62,7 \%$ \\
\hline Cambio en el apetito & $16,6 \%$ & $36,9 \%$ & $46,5 \%$ \\
\hline Cansancio o fatiga & $10,7 \%$ & $26,2 \%$ & $63,1 \%$ \\
\hline
\end{tabular}

Fuente: Elaboración propia a partir del instrumento de Encuesta realizado para la investigación (2020).

En la tabla 7 se identifica que el aislamiento obligatorio preventivo ha propiciado en los estudiantes sentimientos y emociones que afectan su cotidianidad, por ejemplo, se destaca con un aumento del $40 \%$ (con respecto a la condición habitual), los niveles de estrés, cansancio, irritabilidad, desilusión, tensión, pérdida de apetito, indecisión y disconformidad con uno mismo. Asimismo, los estudiantes manifestaron una reducción de al menos el $40 \%$ en el nivel de concentración, de energía e interés en el desarrollo de actividades, así como de las horas de sueño.

\section{Docentes}

\section{Perfil demográfico}

En la tabla 8 se organizaron las características demográficas de los docentes que participaron como informantes. Existe predominio del género masculino, con edades que oscilan entre 30 y 49 años en aproximadamente el $66 \%$ de los casos. En el $57 \%$ de los casos poseen pareja estable mediante una relación legalmente constituida (matrimonio) o de manera informal (unión libre). Respecto a la universidad dónde laboran como docentes de Arquitectura se contó con la participación de diez instituciones educativas distribuidas a lo largo de la región Andina del país (Pasto, Armenia, Manizales, Bogotá, Tunja, Bucaramanga, Pamplona y Cúcuta). Se resalta el caso de un informante Arquitecto en ejercicio quien diligenció el instrumento, pero no labora como docente.

Tabla 8.

Perfil demográfico de los docentes informantes

\begin{tabular}{|c|c|c|c|}
\hline Característica & Niveles de respuesta & Frecuencia & Porcentaje \\
\hline \multirow[t]{3}{*}{ Género } & Masculino & 95 & $70,4 \%$ \\
\hline & Femenino & 40 & $29,6 \%$ \\
\hline & Total & 135 & $100,0 \%$ \\
\hline \multirow[t]{5}{*}{ Edad } & 50 años o más & 39 & $28,9 \%$ \\
\hline & Entre 40 y 49 años & 51 & $37,8 \%$ \\
\hline & Entre 30 y 39 años & 38 & $28,1 \%$ \\
\hline & Entre 20 y 29 años & 7 & $5,2 \%$ \\
\hline & Total & 135 & $100,0 \%$ \\
\hline \multirow[t]{6}{*}{ Estado Civil } & Soltero(a) & 15 & $11,1 \%$ \\
\hline & Casado(a) & 56 & $41,5 \%$ \\
\hline & Unión libre & 21 & $15,6 \%$ \\
\hline & Separado(a) & 15 & $11,1 \%$ \\
\hline & Viudo(a) & 1 & $0,7 \%$ \\
\hline & $\begin{array}{c}\text { Total } \\
\end{array}$ & 135 & $100,0 \%$ \\
\hline Actualmente & Institución Universitaria CESMAG & 7 & $5,1 \%$ \\
\hline
\end{tabular}




\begin{tabular}{|c|c|c|c|}
\hline \multirow[t]{11}{*}{ labora en } & $\begin{array}{c}\text { Universidad Francisco de Paula } \\
\text { Santander }\end{array}$ & 9 & $6,7 \%$ \\
\hline & Universidad Agustiniana & 2 & $1,5 \%$ \\
\hline & Universidad Piloto & 2 & $1,4 \%$ \\
\hline & Universidad del Valle & 1 & $0,7 \%$ \\
\hline & Universidad Católica de Colombia & 2 & $1,4 \%$ \\
\hline & Universidad de los Andes & 9 & $6,6 \%$ \\
\hline & Universidad la Gran Colombia & 71 & $52,6 \%$ \\
\hline & Universidad Nacional de Colombia & 9 & $6,7 \%$ \\
\hline & Universidad Santo Tomás de Aquino & 22 & $16,3 \%$ \\
\hline & Ninguna & 1 & $0,7 \%$ \\
\hline & Total & 135 & $100,0 \%$ \\
\hline
\end{tabular}

Fuente: Elaboración propia a partir del instrumento de Encuesta realizado para la investigación (2020).

\section{Competencias TIC con uso social}

En cuanto al nivel de desempeño que poseen los docentes informantes sobre el uso de redes sociales, se determinó que el $94,1 \%$ afirman que posee conocimientos sobre el funcionamiento de esta, pero de ellos se destaca que el $70,4 \%$ dicen ser competentes en este aspecto. El 83,0\% dicen que han recibido capacitación en el manejo de herramientas o plataformas virtuales.

El $91,9 \%$ de los docentes informantes aseguran dedicar diariamente tiempo al uso de redes sociales, destacándose que el 62,2\% de ellos emplean como máximo dos horas al día a esta actividad, bajo un fin de ocio con el 35,6\% de los casos y como medio para mantenerse actualizado de información en el $57,0 \%$.

Finalmente, se establece que las redes sociales utilizadas con mayor frecuencia corresponden a Instagram, Facebook y Twitter, que concentran el 81,5\% de los casos, con el $34,8 \%, 30,4 \%$ y $16,3 \%$ respectivamente. En la tabla 8 se pueden corroborar los principales hallazgos de esta categoría.

Tabla 9.

Tabla cruzada entre el tiempo diario dedicado a las redes sociales vs Principal razón por la que las utiliza y la red social más utilizada

¿Con qué frecuencia diaria utiliza las redes sociales?

\begin{tabular}{ccccccc} 
& & $\begin{array}{c}\text { Dos horas } \\
\text { o menos }\end{array}$ & $\begin{array}{c}\text { Entre dos y } \\
\text { cinco horas }\end{array}$ & $\begin{array}{c}\text { Más de cinco } \\
\text { horas }\end{array}$ & $\begin{array}{c}\text { No } \\
\text { utilizo }\end{array}$ & Total \\
\hline $\begin{array}{c}\text { Principal } \\
\text { razón por la } \\
\text { que utiliza } \\
\text { las redes } \\
\text { sociales }\end{array}$ & Otra & $6,7 \%$ & & $0,7 \%$ & & $\mathbf{7 , 4 \%}$ \\
\cline { 2 - 7 } & Ocio & $16,3 \%$ & $1,5 \%$ & $12,6 \%$ & $5,2 \%$ & $\mathbf{3 5 , 6 \%}$ \\
\cline { 2 - 7 } & $\begin{array}{c}\text { Para estar } \\
\text { enterado de los } \\
\text { últimos }\end{array}$ & $39,3 \%$ & $5,2 \%$ & $9,6 \%$ & $3,0 \%$ & $\mathbf{5 7 , 0 \%}$ \\
\cline { 2 - 7 } & acontecimientos & & & & & \\
\hline ¿Cuál es la & Total & $\mathbf{6 2 , 2 \%}$ & $\mathbf{6 , 7 \%}$ & $\mathbf{2 3 , 0 \%}$ & $\mathbf{8 , 1 \%}$ & $\mathbf{1 0 0 , 0 \%}$ \\
\hline & Facebook & $\mathbf{2 5 , 2 \%}$ & $1,5 \%$ & $5,9 \%$ & $2,2 \%$ & $\mathbf{3 4 , 8 \%}$ \\
\hline
\end{tabular}




\begin{tabular}{ccccccc} 
red social & Instagram & $18,5 \%$ & $2,2 \%$ & $8,9 \%$ & $0,7 \%$ & $\mathbf{3 0 , 4 \%}$ \\
\cline { 2 - 6 } $\begin{array}{c}\text { que utiliza } \\
\text { con mayor }\end{array}$ & Otra & $8,1 \%$ & $0,7 \%$ & $5,9 \%$ & $3,7 \%$ & $\mathbf{1 8 , 5 \%}$ \\
\cline { 2 - 6 } frecuencia? & Twitter & $10,4 \%$ & $2,2 \%$ & $2,2 \%$ & $1,5 \%$ & $\mathbf{1 6 , 3 \%}$ \\
\cline { 2 - 6 } & Total & $\mathbf{6 2 , 2 \%}$ & $\mathbf{6 , 7 \%}$ & $\mathbf{2 3 , 0 \%}$ & $\mathbf{8 , 1 \%}$ & $\mathbf{1 0 0 , 0 \%}$ \\
\hline
\end{tabular}

Fuente: Elaboración propia a partir del instrumento de Encuesta realizado para la investigación (2020).

\section{Competencias TIC con uso académico}

Con esta categoría se precisa el nivel de apropiación que poseen los docentes informantes sobre las diversas herramientas TIC en el ejercicio de su práctica pedagógica. Así se determinó que el $86,7 \%$ de los docentes informantes afirman que actualmente están desarrollando sus actividades docentes en modalidad no presencial con ayuda de recursos digitales. Por su parte, el $74,1 \%$ de estos, aseguran que antes del proceso de aislamiento obligatorio derivado del COVID-19 habían participado en clases no presenciales.

Al indagar sobre las diversas plataformas o herramientas que se han implementado como recurso de enseñanza por parte del grupo de docentes informantes se recolectó un total de 541 opiniones dado que podían seleccionar más de una opción. De esta manera, se determinó que el $90 \%$ de las opiniones se concentran en seis aplicaciones de las cuales el $57 \%$ son aplicaciones sincrónicas (Hangouts Meet, WhatsApp, Zoom y Google Classroom), mientras que un $33 \%$ son aplicaciones que permiten el envío y almacenamiento de archivos (con limitaciones de capacidad y cantidad) en actividades asincrónicas.

Tabla 10.

Relación de herramientas o plataformas implementadas en el trabajo no presencial

\begin{tabular}{ccc}
\hline Plataforma o herramienta & Frecuencia & Porcentaje \\
\hline Hangouts Meet & 79 & $15 \%$ \\
WhatsApp & 81 & $15 \%$ \\
Correo electrónico & 94 & $17 \%$ \\
Zoom & 96 & $18 \%$ \\
Plataforma Institucional & 89 & $16 \%$ \\
Google Classroom & 48 & $9 \%$ \\
Microsoft Teams & 31 & $6 \%$ \\
Otra & 23 & $4 \%$ \\
\hline Total & $\mathbf{5 4 1}$ & $100,0 \%$ \\
\hline
\end{tabular}

Fuente: Elaboración propia a partir del instrumento de Encuesta realizado para la investigación (2020).

La tabla 11 permite identificar como fortaleza el buen desempeño que dicen tener de las diversas herramientas o plataformas digitales el grupo de docentes informantes $(78,6 \%)$. Asimismo, el 20,7\% aseguran que su desempeño es aceptable, pero a pesar de esta limitante dedican más de dos horas diarias al uso de estos recursos digitales, luego seguramente en muy corto tiempo podrán mejorar sus competencias. 
Tabla 11.

Tabla cruzada entre el tiempo diario dedicado a las plataformas vs su valoración de desempeño en las mismas

¿Con qué frecuencia diaria utiliza las plataformas virtuales con fines educativos?

\begin{tabular}{cccccc}
\cline { 3 - 6 } & \multicolumn{3}{c}{ educativos? } \\
\cline { 2 - 6 } & $\begin{array}{c}\text { Dos horas o } \\
\text { menos }\end{array}$ & $\begin{array}{c}\text { Entre dos y } \\
\text { cinco horas }\end{array}$ & $\begin{array}{c}\text { Más de cinco } \\
\text { horas }\end{array}$ & Total \\
\hline $\begin{array}{c}\text { ¿Considera que su } \\
\text { desempeño en el } \\
\text { manejo de }\end{array}$ & Bueno & $5,9 \%$ & $15,6 \%$ & $57,1 \%$ & $\mathbf{7 8 , 6 \%}$ \\
\cline { 2 - 6 } & $\begin{array}{c}\text { Acepta } \\
\text { ble }\end{array}$ & $3,0 \%$ & $8,1 \%$ & $9,6 \%$ & $\mathbf{2 0 , 7 \%}$ \\
\cline { 2 - 6 } $\begin{array}{c}\text { blataformas } \\
\text { virtuales con fines } \\
\text { educativo es? }\end{array}$ & $\begin{array}{c}\text { Deficie } \\
\text { nte }\end{array}$ & $0,0 \%$ & $0,0 \%$ & $0,7 \%$ & $\mathbf{0 , 7 \%}$ \\
\cline { 2 - 6 } & Total & $\mathbf{8 , 9 \%}$ & $\mathbf{2 3 , 7 \%}$ & $\mathbf{6 7 , 4 \%}$ & $\mathbf{1 0 0 , 0 \%}$ \\
\hline
\end{tabular}

Fuente: Elaboración propia a partir del instrumento de Encuesta realizado para la investigación (2020).

Se logró determinar que el $43,7 \%$ de los docentes informantes dicen que han tenido algunas dificultades en el manejo de estas herramientas, mientras que el 53,3\% dicen que han podido incorporarlas a su actividad pedagógica sin problema alguno.

El $86,7 \%$ de los docentes informantes afirman que han recibido capacitación en cuanto al manejo de estos recursos digitales con fines educativos. Finalmente, en el $75,6 \%$ de los casos dicen que en este proceso de aislamiento obligatorio han realizado actividades tanto sincrónicas como asincrónicas para el desarrollo de sus cátedras, pero el $23,7 \%$ dicen centrar su labor docente sólo en actividades sincrónicas con sus estudiantes.

\section{Afectación emocional}

En la tabla 12 se identifica que el aislamiento obligatorio preventivo ha propiciado en los docentes informantes sentimientos y emociones que afectan su cotidianidad, por ejemplo, se destacan como aspectos negativos el aumento del estrés $(60,7 \%)$, la sensación de cansancio o fatiga $(60,0 \%)$ y una reducción en las horas de sueño $(45,9 \%)$; y como aspectos positivos el aumento del interés por realizar actividades $(49,6 \%)$ y el aumento de la concentración (46,7\%).

Tabla 12.

Relación del grado de afectación emocional derivado del aislamiento preventivo obligatorio

Nivel de afectación

\begin{tabular}{cccc}
\hline Sentimientos & $\begin{array}{c}\text { Ha } \\
\text { reducido }\end{array}$ & $\begin{array}{c}\text { Se mantiene } \\
\text { igual }\end{array}$ & $\begin{array}{c}\text { Ha } \\
\text { aumentado }\end{array}$ \\
\hline Estrés & $11,9 \%$ & $27,4 \%$ & $60,7 \%$ \\
\hline Desilusión & $26,7 \%$ & $49,6 \%$ & $23,7 \%$ \\
\hline Disconformidad consigo mismo & $37,0 \%$ & $53,3 \%$ & $9,6 \%$ \\
\hline Sentimiento de culpa & $38,5 \%$ & $51,1 \%$ & $10,4 \%$ \\
\hline Nivel de concentración & $15,6 \%$ & $37,8 \%$ & $46,7 \%$ \\
\hline Hábitos de sueño & $45,9 \%$ & $46,7 \%$ & $7,4 \%$ \\
\hline Llanto & $37,8 \%$ & $56,3 \%$ & $5,9 \%$ \\
\hline Agitación o tensión & $20,0 \%$ & $44,4 \%$ & $35,6 \%$ \\
\hline
\end{tabular}




\begin{tabular}{cccc} 
Interés por realizar actividades & $13,3 \%$ & $37,0 \%$ & $49,6 \%$ \\
\hline Indecisión & $37,0 \%$ & $49,6 \%$ & $13,3 \%$ \\
\hline Nivel de energía en el desarrollo de actividades & $17,0 \%$ & $54,1 \%$ & $28,9 \%$ \\
\hline Irritabilidad & $31,3 \%$ & $36,3 \%$ & $32,6 \%$ \\
\hline Cambio en el apetito & $17,0 \%$ & $57,0 \%$ & $25,9 \%$ \\
\hline Cansancio o fatiga & $7,4 \%$ & $32,6 \%$ & $60,0 \%$ \\
\hline
\end{tabular}

Fuente: Elaboración propia a partir del instrumento de Encuesta realizado para la investigación (2020).

\section{Discusiones y conclusiones}

Para dar cumplimiento al objetivo perseguido en este artículo, se condensan orientaciones que permiten identificar las necesidades y la realidad planteada con respecto al proceso de educación no presencial de los componentes docente y estudiantil de los programas de Arquitectura en Colombia. Es importante aclarar que los resultados derivados de este estudio y sus posibles conclusiones no pueden ser utilizadas para validar o desacreditar la experiencia de la educación virtual, educación mediada por las TIC o remota.

La información mencionada debe entenderse como el resultado del monitoreo de las actividades educativas y sociales desarrolladas bajo la experiencia de emergencia del aislamiento preventivo obligatorio en respuesta al COVID-19. Esto con el fin de ampliar la información existente para así conocer la realidad del proceso que actualmente se lleva a cabo desde la educación superior y a su vez, obtener insumos que permitan generar estrategias que conlleven a mejorar las prácticas educativas no presenciales.

Sin embargo, y como respuesta a la pandemia del COVID-19, los programas de Arquitectura de las IES nacional han debido realizar adaptaciones de orden pedagógico que les permitan dar cumplimiento al I semestre del 2020; por medio de estrategias pedagógicas no presenciales tales como la utilización de plataformas virtuales y otros elementos tecnológicos. Lo cual coincide con Martín, Hernández y Mendoza (2017) quienes sostienen que un nivel de apropiación en competencia TIC, permite construir espacio o ambiente de aprendizaje basados en la Web.

Esto ha traído como consecuencia un cambio radical en los métodos de enseñanza, sobre todo dentro de las asignaturas de tipo prácticas, a través de las cuales se desarrollan asesorías personalizadas en torno a un proyecto arquitectónico y/o urbano específico de acuerdo a la temática abordada en el semestre académico; además, se debe considerar que en algunas ocasiones estas asignaturas se llevan a cabo bajo grupos de trabajo, para los cuales, la organización de una propuesta de manera no presencial establece un nuevo nivel de complejidad.

De la misma manera, con respecto a las asignaturas teóricas o teórico-prácticas, la no presencialidad ha obligado a los estudiantes (y también a los docentes) a adaptar aplicaciones asociados a lo social o aprender nuevas herramientas de uso académico, en un 
corto periodo de tiempo, para responder a las necesidades del proceso educativo. Por ejemplo, el uso, apropiaciones y prácticas comunicativas de las redes sociales hacia lo educativo (Espinel, Hernández y Rojas, 2020). Esto ha configurado nuevos retos para los programas de Arquitectura, quienes deberán replantear las estrategias pedagógicas y mejorar las competencias TIC de estudiantes y docentes, con el fin de no restar calidad al proceso educativo. Estos cambios y adaptaciones se encuentran influenciado por el medio natural y cultural propio del contexto en el que se desarrolla (Saldarriaga, 1996), en este caso, debido a que existe una intersección entre las TIC, educación y pedagogía (Parra, 2010), que soporta los estudios no presenciales.

A lo mencionado, hay que sumar el colapso frecuente del servicio de internet (por el aumento de personas conectadas de manera simultánea), así como a la condición socioeconómica de algunos estudiantes para tener conectividad, que en ocasiones impiden realizar actividades sincrónicas de asesoría y acompañamiento, lo que dificulta llevar un proceso educativo normal como el que se realiza en el modelo presencial.

Por otra parte, como un hallazgo del estudio, los docentes encuestados afirman que, debido al aislamiento social y al desarrollo de actividades no presenciales, han desarrollado un aumento del interés por realizar actividades, pero a su vez han experiementado un aumento en los niveles de estrés derivado de las nuevas prácticas pedagógicas adoptadas. Esto es contrario a investigaciones como las de Rodríguez-Martínez et al. (2018)quienes identificaron que la ansiedad y la depresión son predictores del estrés laboral en docentes de educación superior.

Con respecto a los estudiantes, se resaltan las manifestaciones asociadas al aumento del estrés, cansancio y fatiga; así como en lo referente a las afectaciones a nivel emocional, en los hábitos de sueño, alimentación y concentración. Estos, afirman que los docentes, en muchos casos asignan actividades sin dosificar el tiempo que demanda su realización. Lo anterior, sumado al hecho de que no recibieron instrucción en el uso de los recursos digitales para fines académicos, se convierten en elementos que generan estrés, ansiedad y depresión. Estos sentimientos que están presentes en los estudiantes afectan su salud mental, tal como se menciona en las investigaciones de Montoya, Gutiérrez y Toro (2010) y Tijerina et al. (2019).

Finalmente, este estudio proporciona información para entender los cambios a los que actualmente se enfrentan los docentes y los estudiantes con respecto al desarrollo de su proceso de educación superior, en este caso específico para el programa de Arquitectura. Además, establece datos y hallazgos relevantes con respecto a los aspectos que deben ser tenidos en cuenta desde la orientación institucional, con el fin de ayudar a solventar las inquietudes, problemáticas y las inconformidades generadas a partir del aislamiento preventivo obligatorio por COVID-19. De esta manera, se resalta que uno de los aspectos relevantes dentro del estudio realizado a los componentes: docente y estudiantes de los programas de Arquitectura a nivel nacional, corresponde a la puesta en marcha de procesos 
de sensibilización y formación pedagógica bajo herramientas TIC de orden académico tanto para los docentes como para los estudiantes, con el fin de generar en la prestación y en la toma del servicio educativo, así como de disminuir los efectos negativos asociados al estrés, los cambios en los hábitos del sueño y la ansiedad, entre otros.

\section{Referencias}

Aparici, R. y Silva, M. (2012). Pedagogía de la interactividad. Comunicar. 38(19), 51-58. https://doi.org/10.3916/C38-2012-02-05

Brooks, S. K., Webster, R. K., Smith, L. E., Wooland, L., Wessely, S., Greenberg, N., \& Rubin, G. J. (2020). The psychological impact of quarantine and how to reduce it: rapide review of the evidence. The Lancet, (395), 912-920. https://doi.org/10.1016/S0140$6736(20) 30460-8$

Cauchemez, S., Ferguson, N. M., Wachtel, C., Tegnell, A., Saour, G., Duncan, B., \& Nicoll, A. (2009). Closure of schools during an influenza pandemic. Lancet infectious diseases, 8(9), 473-481. https://doi.org/10.1016/S1473-3099(09)70176-8

Dirección de comunicaciones de la Fundación Universitaria del Área Andina. (2020). Virtualidad un antídoto de la educación en tiempos de coronavirus. Asociación Colombiana de Universidades (ASCUN). https://www.ascun.org.co/noticias/detalle/virtualidad-un-antidoto-de-la-educacionen-tiempos-de-coronavirus-273

Gamboa, A., Hernández, C. y Prada, R. (2018). Práctica pedagógica y competencias TIC. Saber, Ciencia y Libertad, 13(1), 258-274. https://doi.org/10.18041/23823240/saber.2018v13n1.2090

Espinel, G., Hernández, C. y Rojas, J. (2020). Usos, apropiaciones y nuevas prácticas comunicativas de los usuarios adolescentes de facebook. Saber, Ciencia y Libertad, 15(1), 280-296. https://doi.org/10.18041/2382-3240/saber.2020v15n1.6316

González, V., González, N., Gómez, C., Palacio, C., Gómez, A. y Franco, O. (2020). Proyecciones de impacto de la pandemia COVID-19 en la población colombiana, según medidas de mitigación. Datos preliminares de modelos epidemiológicos para el periodo del 18 de marzo al 18 de abril de 2020. Revista de salud pública, 22(1), http://dx.doi.org/10.15446/rsap.v22.85789

Hernández, C., Arévalo, M. y Gamboa, A. (2016). Competencias TIC para el desarrollo profesional docente en educación básica. Praxis \& Saber, Л14), 41 - 69. https://doi.org/10.19053/22160159.5217 
Hernández, C., Ayala, E. y Gamboa, A. (2016). Modelo de competencias TIC para docentes: Una propuesta para la construcción de contextos educativos innovadores y la consolidación de aprendizajes en educación superior. Katharsis, (22), 221-265. https://doi.org/10.25057/25005731.821

Hernández, C., Prada, R. y Ramírez, P. (2018). Perspectivas actuales de los docentes de educación básica y media acerca de la aplicación de las competencias tecnológicas en el aula. Revista Espacios, 49(43), 19. http://www.revistaespacios.com/a18v39n43/a18v39n43p19.pdf

Hernández, R., Fernández, C. y Baptista, P. (2010). Metodología de la investigación. México: McGraw Hill

Instituto Colombiano para la Evaluación de la Educación - ICFES. (2019a). Guía de orientación Saber Pro 2019: Modulo de proyecto de arquitectura. https://www.icfes.gov.co/documents/20143/1518930/Guia+de+modulo+de+proye cto+de+arquitectura+2019.pdf/6b909610-a007-63da-5a72-55ea7e2cc360

Instituto Colombiano para la Evaluación de la Educación - ICFES. (2019b). Guía Orientación Saber Pro 2019: módulos competencias genéricas. https://www.icfes.gov.co/documents/20143/1518930/Guia+de+orientacion+modul os+de+competencias+genericas+saber+pro+2019.pdf/3fe99e8b-229a-c4e8-3aedf4b719460c51

Instituto Colombiano para la Evaluación de la Educación - ICFES. (2019c). Marco de referencia del módulo de estudio proyectual. Saber Pro. https://www.icfes.gov.co/documents/20143/1525601/Marco+de+referencia++modulo+de+estudio+proyectual.pdf/c13a46e2-9e8a-b8fe-ede1-95ff56134023

Instituto de Salud Carlos III - Sistema de vigilancia de la gripe en España. (2018, 16 de abril). Las vacaciones escolares contribuyen a mitigar la gripe. Asociación Española de Pediatría. https://vacunasaep.org/profesionales/noticias/gripe-vacacionesescolares

Instituto Internacional para la educación superior en América Latina y el Caribe - IESALC. (2020, 2 de abril). EL CORONAVIRUS COVID-19 y la educación superior: Impacto y recomendaciones. https://www.iesalc.unesco.org/2020/04/02/el-coronavirus-covid19-y-la-educacion-superior-impacto-y-recomendaciones/

Instituto Nacional de Salud Colombia (2007, 25 de junio). Informe epidemiológico nacional. https://www.ins.gov.co

Instituto Nacional de Salud Colombia. (2020, 19 de mayo). Coronavirus (Covid-2019) en Colombia. https://www.ins.gov.co/Noticias/Paginas/Coronavirus.aspx 
Leybold-Johnson, I. (2020, 13 de abril). Educación en tiempos de pandemia. Coronavirus: efectos a largo plazo del cierre de las escuelas. Swissinfoch. https://www.swissinfo.ch/spa/sociedad/educaci\%C3\%B3n-en-tiempos-depandemia-_coronavirus--efectos-a-largo-plazo-del-cierre-de-las-escuelas/45680314

Linne, J. (2014). Usos comunes de Facebook en adolescentes de distintos sectores sociales en la Ciudad de Buenos Aires. Comunicar, 43(22), 189-197. https://doi.org/10.3916/C43-2014-19

Mahase, E. (2020). Covid-19: WHO declares pandemic because of "alarming levels" of spread, severity, and inaction. BMJ, (368), m1036. https://doi.org/10.1136/bmj.m1036

Martín, M., Hernández, C. y Mendoza, S. (2017). Ambientes de aprendizaje basados en herramientas web para el desarrollo de competencias TIC en la docencia. Perspectivas, 2(1). 97-104. https://doi.org/10.22463/25909215.1282

Ministerio de Educación Nacional de Colombia. (17 de marzo de 2020). Circular 021 de 2020 Orientaciones para el desarrollo de procesos de planeación pedagógica y trabajo académico en casa como medida para la prevención de la propagación del Coronavirus (COVID-19), así como para el manejo del personal docente, directivo docente y administrativo del sector educación. https://www.mineducacion.gov.co/1759/articles-394115_recurso_1.pdf

Ministerio de Justicia y del Derecho de la República de Colombia. (2020, 14 abril). Decreto Legislativo 546 de 2020 Por medio del cual se adoptan medidas para sustituir la pena de prisión y la medida de aseguramiento de detención preventiva en establecimientos penitenciarios y carcelarios por la prisión domiciliaria y la detención domiciliaria transitorias en el lugar de residencia a personas que se encuentran en situación de mayor vulnerabilidad frente al COVID-19, y se adoptan otras medidas para combatir el hacinamiento carcelario y prevenir y mitigar el riesgo de propagación, en el marco del Estado de Emergencia Económica, Social y Ecológica. https://dapre.presidencia.gov.co/normativa/normativa/DECRETO\%20546\%20DEL \%2014\%20DE\%20ABRIL\%20DE\%202020.pdf

Ministerio de Salud y Protección de la República de Colombia. (2020,12 de marzo). Resolución 385 de 2020 Por la cual se declara la emergencia sanitaria por causa del coronavirus COVID-19 y se adoptan medidas para hacer frente al virus. https://www.minsalud.gov.co/sites/rid/Lists/BibliotecaDigital/RIDE/DE/DIJ/resoluci on-385-de-2020.pdf

Ministerio de salud y protección social de la República de Colombia. (2020, 12 abril). Decreto Legislativo 538 de 2020 Por el cual se adoptan medidas en el sector salud, para contener y mitigar la pandemia de COVID-19 y garantizar la prestación de los 
servicios de salud, en el marco del Estado de Emergencia Económica, Social y Ecológica.

https://dapre.presidencia.gov.co/normativa/normativa/DECRETO\%20538\%20DEL \%2012\%20DE\%20ABRIL\%20DE\%202020.pdf

Ministerio de Salud y Protección Social de la República de Colombia. (2020, 18 de marzo). Resolución 464 de 2020 Por la cual se adopta la medida sanitaria obligatoria de aislamiento preventivo, para proteger a los adultos mayores de 70 años. https://www.minsalud.gov.co/sites/rid/Lists/BibliotecaDigital/RIDE/DE/DIJ/resoluci on-464-de-2020.pdf

Ministerio de Transporte de la República de Colombia. (2020, 20 de marzo). Decreto 439 de 2020 Por el cual se suspende el desembarque con fines de ingreso o conexión en territorio colombiano, de pasajeros procedentes del exterior, por vía aérea. https://dapre.presidencia.gov.co/normativa/normativa/DECRETO\%20439\%20DEL \%2020\%20DE\%20MARZO\%20DE\%202020.pdf

Ministerio del Interior de la República de Colombia (2020, 24 de abril). Decreto 593 de 2020 Por el cual se imparten instrucciones en virtud de la emergencia sanitaria generada por la pandemia del Coronavirus COVID-19, y el mantenimiento del orden público. Diario Oficial de la nación. https://dapre.presidencia.gov.co/normativa/normativa/DECRETO\%20593\%20DEL \%2024\%20DE\%20ABRIL\%20DE\%202020.pdf

Ministerio del Interior de la República de Colombia (2020, Colombia, 13 de marzo). Decreto 402 de 2020 Por el cual se adoptan medidas para la conservación del orden público. https://dapre.presidencia.gov.co/normativa/normativa/DECRETO\%20402\%20DEL \%2013\%20DE\%20MARZO\%20DE\%202020.pdf

Ministerio del Interior de la República de Colombia. (2020, 22 de marzo). Decreto 457 Por el cual se imparten instrucciones en virtud de la emergencia sanitaria generada por la pandemia del Coronavirus COVID-19 y el mantenimiento del orden público. https://dapre.presidencia.gov.co/normativa/normativa/DECRETO\%20457\%20DEL \%2022\%20DE\%20MARZO\%20DE\%202020.pdf

Ministerio del Interior de la República de Colombia. (2020, 8 de abril). Decreto 531 de 2020 Por el cual se imparten instrucciones en virtud de la emergencia sanitaria generada por la pandemia del Coronavirus COVID-19, y el mantenimiento del orden público. https://dapre.presidencia.gov.co/normativa/normativa/DECRETO\%20531\%20DEL \%208\%20DE\%20ABRIL\%20DE\%202020.pdf

Ministerio del Trabajo de la República de Colombia. (2020, 24 de febrero). Circular 017 de 2020 Lineamientos mínimos a implementar de promoción y prevención para la preparación, respuesta y atención de casos de enfermedad por covid-19 (antes 
denominado

coronavirus).

https://www. mintrabajo.gov.co/documents/20147/0/Circular+0017.pdf/05096a91e470-e980-2ad9-775e8419d6b1?t=1582647828087

Montoya, L., Gutiérrez, J. y Toro, B. (2010). Depresión en estudiantes universitarios y su asociación con el estrés académico. CES Medicina, 24(1). https://revistas.ces.edu.co/index.php/medicina/article/view/1011

Organización Mundial de la Salud - OMS. (2020a, febrero 19). Alocución de apertura del director general de la OMS en rueda de prensa para las misiones diplomáticas sobre la Covid-19. OMS. https://www.who.int/es/dg/speeches/detail/who-directorgeneral-s-opening-remarks-at-the-mission-briefing-on-covid-19

Organización Mundial de la Salud - OMS. (2020b, mayo 20). Coronavirus Disease (COVID19) DashboardOMS. https://covid19.who.int

Organización Mundial de la Salud - OMS. (2020c). Orientaciones para el público. OMS. https://www.who.int/es/emergencies/diseases/novel-coronavirus-2019/advice-forpublic

Organización Panamericana de la Salud - OPS. (2020). Similitudes y diferencias entre la $\begin{array}{lllll}\text { COVID-19 } y & \text { la } & \text { gripe. }\end{array}$ https://www.paho.org/hq/index.php?option=com_content\&view=article\&id=15760 :similarities-and-differences-covid-19-and-influenza\&Itemid=1926\&lang=es

Pan American Health Organization - PAHO. (2020, mayo 20). Cumulative COVID-19 cases reported by countries and territories in the Americas. PAHO. https://who. maps.arcgis.com/apps/webappviewer/index.html?id=2203b04c3a5f48 6685a15482a0d97a87\&extent $=-17277700.8881 \% 2 C-1043174.5225 \% 2 C-$ $1770156.5897 \% 2 C 6979655.9663 \% 2 C 102100$

Parra, C. (2010). Intersecciones entre las TIC, la educación y la pedagogía en Colombia: hacia una reconstrucción de múltiples miradas. Nómadas, (33), 215-225. http://nomadas.ucentral.edu.co/index.php/inicio/16-procesos-de-colonizacionresistencia-y-descolonizacion-nomadas-33/181-intersecciones-entre-las-tic-laeducacion-y-la-pedagogia-en-colombia-hacia-una-reconstruccion-de-multiplesmiradas

Presidencia de la República de Colombia. (17 de marzo de 2020). Decreto 417 de 2020 Por el cual se declara un Estado de Emergencia Económica, Social y Ecológica en todo el territorio Nacional. Diario Oficial de la nación. https://dapre.presidencia.gov.co/normativa/normativa/DECRETO\%20417\%20DEL \%2017\%20DE\%20MARZO\%20DE\%202020.pdf 
Presidencia de la República de Colombia. (6 de mayo de 2020). Decreto 637 de 2020Por el cual se declara un Estado de Emergencia Económica, Social y Ecológica en todo el territorio Nacional. Diario Oficial de la nación. https://dapre.presidencia.gov.co/normativa/normativa/DECRETO\%20637\%20DEL \%206\%20DE\%20MAYO\%20DE\%202020.pdf

Rodríguez-Martínez, M., Tovalin-Ahumada, J. H., Gil-Monte, P. R., Salvador-Cruz, J. y AcleTomasini, G. (2018). Trabajo emocional y estresores laborales como predictores de ansiedad y depresión en profesores universitarios mexicanos. Informació psicològica, (115), 93-106. http://dx.medra.org/10.14635/IPSIC.2018.115.11

Saldarriaga, A. (1996). Aprender Arquitectura. Un manual de supervivencia. Bogotá: Corona.

Saldarriaga, A., Mateus, J. y Pinzón, J. A. (2011). La enseñanza de la arquitectura en Colombia. Estado Actual. Bogotá: ACFA.

Summers, G. (1976). Medición de actitudes. México: Trillas.

Tijerina, L., González, E., Gómez, M., Cisneros, M., Rodríguez, K. y Ramos, E. (2019). Depresión, ansiedad y estrés en estudiantes de nuevo ingreso a la educación superior. RESPYN Revista Salud Pública y Nutrición, 17(4), 41-47. https://doi.org/10.29105/respyn17.4-5

UNESCO. (2020a). Consecuencias negativas del cierre de las escuelas. UNESCO. https://es.unesco.org/covid19/educationresponse/consecuencias

UNESCO. (2020b). Interrupción educativa y respuesta al Covid-19. UNESCO. https://es.unesco.org/covid19/educationresponse

UNICEF. (2020). COVID-19: fuerte pérdida de ingresos, dificultades en la compra de alimentos y aprobación del aislamiento social preventivo. UNESCO. https://www.unicef.org/argentina/comunicados-prensa/covid-19-unicef-encuestapercepcion-poblacion

Universidad Francisco de Paula Santander - UFPS. (2018). Informe de los estándares de calidad para la renovación de registro calificado de Arquitectura. Cúcuta: UFPS.

Vásquez, M., Ortiz, M., Álvarez, H., Pérez, M., Arias, E. y Bergamaschi, A. (2020). Cierre de escuelas: el desafío que el COVID-19 impuso a los sistemas educativos de ALC. https://blogs.iadb.org/educacion/es/cierredeescuelas/

Wallinga, J., Teunis, P., \& Kretzschmar, M. (2006). Using data on social contacts to estimate age-specific transmission parameters for respiratory-spread infectious agents. American Journal of Epidemiology, 164(10), 936-944. https://doi.org/10.1093/aje/kwj317 